Article

\title{
Axiomatic and Dynamic Results for Power Indexes under Symmetry
}

\author{
Ruey-Rong Huang ${ }^{1}$ and Yu-Hsien Liao ${ }^{2, *}$ \\ 1 General Education Center, Chung-Jen Junior College of Nursing, Health Sciences and Management, \\ Chia-Yi 62241, Taiwan; m021@cjc.edu.tw \\ 2 Department of Applied Mathematics, National Pingtung University, Pingtung 90039, Taiwan \\ * Correspondence: twincos@ms25.hinet.net or twincos@mail.nptu.edu.tw; Tel.: +886-958-631-010
}

check for

updates

Citation: Huang, R.-R.; Liao, Y.-H. Axiomatic and Dynamic Results for Power Indexes under Symmetry. Axioms 2021, 10, 345. https://doi. org/10.3390/axioms10040345

Academic Editors: Humberto Bustince and Oscar Castillo

Received: 26 October 2021

Accepted: 15 December 2021

Published: 16 December 2021

Publisher's Note: MDPI stays neutral with regard to jurisdictional claims in published maps and institutional affiliations.

Copyright: (C) 2021 by the authors. Licensee MDPI, Basel, Switzerland. This article is an open access article distributed under the terms and conditions of the Creative Commons Attribution (CC BY) license (https:// creativecommons.org/licenses/by/ $4.0 /)$.

\begin{abstract}
Symmetry exists in a multitude of phenomena in varying forms. The main aim of this article is to analyze the plausibility of the equal allocation non-separable costs, the efficient Banzhaf-Owen index and the efficient Banzhaf-Coleman index from the perspective of symmetry. First, based on the difference between "participation processes" and "allocating results", different forms of symmetry are proposed. Next, building on these forms of symmetry, axiomatic results are put forth for the three power indexes, whereby the plausibility of the three power indexes is analyzed. Finally, on the basis of these different forms of symmetry and related axiomatic results, this article introduces different dynamic processes to analyze how an initial allocation result approaches the results derived from the three power indexes through dynamically modification.
\end{abstract}

Keywords: power index; axiomatic and dynamic result; symmetry

MSC: 91A; 91B

\section{Introduction}

Three power indexes are considered in this article, the equal allocation non-separable costs (EANSC), the efficient Banzhaf-Coleman index (EBCI) and the efficient BanzhafOwen index (EBOI). The Banzhaf-Coleman index originally defined in the issue of voting problems by Banzhaf [1]. And later on generalized to arbitrary issues by Owen [2], it is named as Banzhaf-Owen index. On the other hand, Ransmeier [3] introduced the EANSC to analyze the optimum yield for dams operated by the Tennessee Valley Authority. Inspired by the notion of the EANSC, Hwang and Liao [4,5] introduced the EBCI and the EBOI respectively. Under the notion of the EANSC (EBCI, EBOI), participants first receive their marginal utilities (entire marginal utilities, average marginal utilities) and further distribute the remaining utilities equally. Moulin [6] showed that the EANSC is the only power index satisfying efficiency, equal treatment for equal, covariance and consistency. Further, Hwang [7] and Hwang and Liao [4,5] characterized the EBCI and the EBOI by means of the standardness property due to Hart and Mas-Colell [8]. Related researches could be studied in Dubey and Shapley [9], Lehrer [10], Haller [11], Brink and Laan [12], and so on.

Symmetry is defined in different forms and endowed with relative senses in different fields. In many studies on the game theory, the symmetry of allocation is often discussed, analyzed and applied from different perspectives, which involve many aspects, such as symmetry of benefits, resources, and power. For example, the contribution of each department in a company cannot be assessed by one uniform standard, but rather should be evaluated based on the symmetry of the contribution ratio of each department in relation to its business. Maschler and Owen [13], Moulin [6] and Peleg [14] conducted axiomatic characterizations of the Shapley value, the EANSC and the Prekernal respectively by considering this notion of symmetry. An illustrative case is that, in a legislative institution, 
the number of legislative representatives from each region should also be assessed in proportion to the population density, industrial value, and regional attributes of each region. Shapley [15] and Myerson [16] also conducted axiomatic characterizations of the Shapley value by considering this notion of symmetry.

Based on the above statement, the motivation of this study can be summarized:

- Can unique symmetry axioms be derived from different behavioral models and then be applied to conduct axiomatic characterizations of these power indexes?

The main results of this article are as follows.

1. In an interactive system or model, disparities may occur among participants and related groups as a result of multiple interactive factors. For example, in a production project of a for-profit organization, its participating members and groups will generate benefits during the participation process, and all members and groups will be remunerated at the end of the project. At this point, discrepancies may arise in the benefits generated and remunerations acquired by the members or groups. Positive and negative differences respectively represent complaints and satisfaction of the members or groups. Unlike past symmetry concepts, this article would like to develop a set of excess concepts for allocation based on the differences between "process involvement" and "allocating outcomes". Then, the excess concepts would be combined with participatory behaviors to define three different symmetry axioms.

2. Further, the allocative efficiency of resources would combined with different symmetry axioms to axiomatically characterize three power indexes.

3. Finally, this article would used the power indexes, each with a different set of symmetry axioms, to produce three different modification functions and related dynamic processes. To be precise and brief, these dynamic processes apply the functions modification to gradually adjust the randomly efficient outcomes and to approach a state of symmetry. According to the axiomatic results, an efficient power index that approximates one of the symmetrical states implies that it approximates all three power indexes.

\section{Preliminaries}

Let $U P$ be a finite and non-empty collection of total participants. A coalition is a non-empty collection of $U P$. A transferable-utility (TU) game is denoted by $(P, C)$, where $P$ is a coalition and $C$ is a mapping such that $C: 2^{P} \longrightarrow \mathbb{R}$ and $C(\varnothing)=0$. Denote the class of all TU games by TG. An index on TG is a mapping $\kappa$ which associates with each TU game $(P, C) \in T G$ an element $\kappa(P, C)$ of $\mathbb{R}^{P}$. An index $\kappa$ satisfies efficiency (EFF) if for all $(P, C) \in T G, \sum_{i \in P} \kappa_{i}(P, C)=C(P)$. EFF states that all participants allot whole utility entirely.

\section{Definition 1.}

- The equal allocation non-separable costs (EANSC), $\overline{\theta^{M}}$, is the index on TG which associates with $(P, C) \in T G$ and all participants $i \in P$ the value

$$
\overline{\theta_{i}^{M}}(P, C)=\theta_{i}^{M}(P, C)+\frac{1}{|P|}\left[C(P)-\sum_{k \in P} \theta_{k}^{M}(P, C)\right],
$$

where $\theta_{i}^{M}(P, C)=C(P)-C(P \backslash\{i\})$ is the marginal index of $i$. The marginal index of $i$ could be treated as the marginal contribution of $i$ in the grand coalition P. Under the index $\overline{\theta^{M}}$, all participants receives their marginal indexes respectively, and allocate the rest of utility simultaneously.

- The efficient Banzhaf-Owen index (EBOI), $\overline{\theta^{B O}}$, is the index on TG which associates with $(P, C) \in T G$ and all participants $i \in P$ the value

$$
\overline{\theta_{i}^{B O}}(P, C)=\theta_{i}^{B O}(P, C)+\frac{1}{|P|}\left[C(P)-\sum_{k \in P} \theta_{k}^{B O}(P, C)\right]
$$


where $\theta_{i}^{B O}(P, C)=\sum_{\substack{S \subseteq P \\ i \in S}}[C(S)-C(S \backslash\{i\})]$ is the Banzhaf-Owen index of $i$. The BanzhafOwen index of $i$ could be treated as the all marginal contributions of $i$ among all participated coalition. Since the Banzhaf-Owen index violates EFF, Hwang and Liao [5] proposed the efficient Banzhaf-Owen index $\overline{\theta^{B O}}$. Under the index $\overline{\theta^{B O}}$, all participants receives their Banzhaf-Owen indexes respectively, and allocate the rest of utility simultaneously.

- The efficient Banzhaf-Coleman index (EBCI), $\overline{\theta^{B C}}$, is the index on $T G$ which associates with $(P, C) \in T G$ and all participants $i \in P$ the value

$$
\overline{\theta_{i}^{B C}}(P, C)=\theta_{i}^{B C}(P, C)+\frac{1}{|P|}\left[C(P)-\sum_{k \in P} \theta_{k}^{B C}(P, C)\right],
$$

where $\theta_{i}^{B C}(P, C)=\frac{1}{2^{|P|-1}} \cdot \sum_{\substack{S \subseteq P \\ i \in S}}[C(S)-C(S \backslash\{i\})]$ is the Banzhaf-Coleman index of $i$. The Banzhaf-Coleman index of $i$ could be treated as the average marginal contribution of $i$ among all participated coalition. Since the Banzhaf-Coleman index violates EFF, Hwang and Liao [4] proposed the efficient Banzhaf-Coleman index $\overline{\theta^{B C}}$. Under the index $\overline{\theta^{B C}}$, all participants receives their Banzhaf-Coleman indexes respectively, and allocate the rest of utility simultaneously.

It is shown that these indexes satisfies EFF simultaneously.

Example 1. In the following, a practical example is given below to illustrate the three indexes and related applications.

1. In a legislative institution, some power appraisal bodies conduct evaluations based on differences in the influence of legislative representatives as to their participation in the overall sitting, known as the marginal index by Definition 1. After the influence of each legislative representative is evaluated, the remaining power is allocated to all the representatives, that is, the EANSC in Definition 1.

2. Some power appraisal bodies conduct evaluations based on differences in the overall influence of legislative representatives as to their participation in a bill committee in relation to their professionalism, known as the Banzhaf-Owen index by Definition 1. After the overall influence of each legislative representative is evaluated, the remaining power is allocated to all the representatives, that is, the EBOI in Definition 1.

3. Some power appraisal bodies conduct evaluations based on differences in the average influence of legislative representatives as to their participation in a bill committee in relation to their professionalism, known as the Banzhaf-Coleman index by Definition 1. After the average influence of each legislative representative is evaluated, the remaining power is allocated to all the representatives, that is, the EBCI in Definition 1.

Subsequently, some existing axioms and related axiomatic results for these three indexes are provided. For each of these efficient indexes, there is a corresponding definition of reduced game as follows. Given an index $\kappa,(P, C) \in T G$ and $S \subseteq P$.

- The complement-reduced game $\left(S, C_{S, K}^{\mathrm{crg}}\right)$ is defined by for all $T \subseteq S$,

$$
C_{S, \kappa}^{c r g}(T)= \begin{cases}C\left(T \cup S^{c}\right)-\sum_{i \in S^{c}} \kappa_{i}(P, C) & \text { if } T \neq \varnothing, \\ 0 & \text { if } T=\varnothing .\end{cases}
$$


- $\quad$ The efficiency-sum-reduced game $\left(S, C_{S, K}^{\text {esrg }}\right)$ is defined by for all $T \subseteq S$,

$$
C_{S, \kappa}^{e s r g}(T)= \begin{cases}C(P)-\sum_{i \in S^{c}} \kappa_{i}(P, C) & \text { if } T=S, \\ \sum_{Q \subseteq S^{c}}\left[C(T \cup Q)-\sum_{i \in Q} \kappa_{i}(P, C)\right] & \text { if } T \neq \varnothing, S, \\ 0 & \text { if } T=\varnothing .\end{cases}
$$

- The efficiency-average-reduced game $\left(S, C_{S, K}^{\text {earg }}\right)$ is defined by for all $T \subseteq S$,

$$
C_{S, K}^{e a r g}(T)= \begin{cases}C(P)-\sum_{i \in S^{c}} \kappa_{i}(P, C) & \text { if } T=S, \\ \frac{1}{2^{S^{c} \mid}} \cdot \sum_{Q \subseteq S^{c}}\left[C(T \cup Q)-\sum_{i \in Q} \kappa_{i}(P, C)\right] & \text { if } T \neq \varnothing, S, \\ 0 & \text { if } T=\varnothing .\end{cases}
$$

For each of these reduced games, there is a corresponding consistency as follows. Let $\kappa$ be an index.

- $\quad \kappa$ satisfies complement-consistency (CCON) if for all $(P, C) \in T G$, for all $S \subseteq P$ and for all $i \in S, \kappa_{i}\left(S, C_{S, K}^{\text {crg }}\right)=\kappa_{i}(P, C)$.

- $\quad \kappa$ satisfies efficiency-sum-consistency (ESCON) if for all $(P, C) \in T G$, for all $S \subseteq P$ and for all $i \in S, \kappa_{i}\left(S, C_{S, \kappa}^{\text {esrg }}\right)=\kappa_{i}(P, C)$.

- $\quad \kappa$ satisfies efficiency-average-consistency (EACON) if for all $(P, C) \in T G$, for all $S \subseteq P$ and for all $i \in S, \kappa_{i}\left(S, C_{S, \kappa}^{\text {earg }}\right)=\kappa_{i}(P, C)$.

Hwang [7] and Hwang and Liao [4] introduced three extended analogues of the standard for games due to Hart and Mas-Colell [8]. Let $\kappa$ be an index.

- $\quad \kappa$ satisfies complement-standardness (COMS) if for all $(P, C) \in T G$ with $|P| \leq 2$, $\kappa(P, C)=\overline{\theta^{M}}(P, C)$.

- $\quad \kappa$ satisfies efficient-Banzhaf-Owen standardness (EBOS) if for all $(P, C) \in T G$ with $|P| \leq 2, \kappa(P, C)=\overline{\theta^{B O}}(P, C)$.

- $\quad \kappa$ satisfies efficient-Banzhaf-Coleman standardness (EBCS) if for all $(P, C) \in T G$ with $|P| \leq 2, \kappa(P, C)=\overline{\theta^{B C}}(P, C)$.

Hwang [7] and Hwang and Liao [4] characterized these power indexes by adopting related properties of consistency and standardness as follows.

- The index $\overline{\theta^{M}}$ is the only index satisfying COMS and CCON.

- The index $\overline{\theta^{B O}}$ is the only index satisfying EBOS and ESCON.

- The index $\overline{\theta^{B C}}$ is the only index satisfying EBCS and EACON.

\section{Symmetry and Axiomatic Results}

In this section, several types of symmetry axiom would be introduced to characterize these indexes. In the following, symmetry refers to the difference between the "participating process" and "allocating results" perceived by participants and related groups.

Let $(P, C) \in T G, S \subseteq P$ and $\kappa$ be an index. Define that $\kappa_{S}(P, C)$ for the restriction of $\kappa(P, C)$ to $S$ and $|\kappa(P, C)|_{S}=\sum_{i \in S} \kappa_{i}(P, C)$. The excess of $S \subseteq P$ at $\kappa$ is

$$
\operatorname{ex}(S, C, \kappa(P, C))=C(S)-|\kappa(P, C)|_{S} .
$$

Based on the consideration of balancing the differences among all members and groups with respect to different participation behaviors, one could identify three different types of symmetry axiom and provides specific definitions for each type. Let $\kappa$ be an index.

- $\quad \kappa$ satisfies complement symmetry under excess (CSE) if for all $(P, C) \in T G$ and for all $i, j \in P$,

$$
\operatorname{ex}(P \backslash\{i\}, C, \kappa(P, C))=\operatorname{ex}(P \backslash\{j\}, C, \kappa(P, C)) .
$$


The complement symmetry under excess states that when two participants do not participate in the largest possible environment, the excesses derived from the power index should be identical. Based on Example 1, in a legislative institution, this type of symmetry focuses on the need to balance the difference between any two legislative representatives as to their respective participation in the overall sitting.

- $\kappa$ satisfies sum symmetry under excess (SSE) if for all $(P, C) \in T G$ and for all $i, j \in P$,

$$
\sum_{S \subseteq P \backslash\{i, j\}} \operatorname{ex}(S \cup\{i\}, C, \kappa(P, C))=\sum_{S \subseteq P \backslash\{i, j\}} \operatorname{ex}(S \cup\{j\}, C, \kappa(P, C)) .
$$

The sum symmetry under excess states that when two participants drop out of all participating environments respectively, the sum of the excesses derived from the allocation method should be identical. Based on Example 1, in a legislative institution, this type of symmetry focuses on the need to balance the total difference between any two legislative representatives as to their respective participation in all professional committee bills.

- $\quad \kappa$ satisfies average symmetry under excess (ASE) if for all $(P, C) \in T G$ and for all $i, j \in P$,

$$
\sum_{S \subseteq P \backslash\{i, j\}} \operatorname{ex}\left(S \cup\{i\}, C, \frac{\kappa(P, C)}{2^{|P|-1}}\right)=\sum_{S \subseteq P \backslash\{i, j\}} \operatorname{ex}\left(S \cup\{j\}, C, \frac{\kappa(P, C)}{2^{|P|-1}}\right) .
$$

The average symmetry under excess states that when two participants drop out of all participating environments respectively, the average excesses derived from the allocation method should be identical. Based on Example 1, in a legislative institution, this type of symmetry focuses on the need to balance the average difference between any two legislative representatives as to their respective participation in all professional committee bills.

The following results present that the three index satisfy three different types of symmetry axiom respectively.

\section{Lemma 1.}

1. The index $\overline{\theta^{M}}$ satisfies CSE.

2. The index $\overline{\theta^{B O}}$ satisfies ASE.

3. The index $\overline{\theta^{B C}}$ satisfies SSE.

Proof. To verify (1), let $(P, C) \in T G$ and $x=\overline{\theta^{M}}(P, C)$. For all $i, j \in P$,

$$
\begin{aligned}
& \operatorname{ex}(P \backslash\{i\}, C, x)-\operatorname{ex}(P \backslash\{j\}, C, x) \\
= & {\left[C(P \backslash\{i\})-|x|_{P \backslash\{i\}}\right]-\left[C(P \backslash\{j\})-|x|_{P \backslash\{j\}}\right] } \\
= & {\left[C(P \backslash\{i\})-x_{j}\right]-\left[C(P \backslash\{j\})-x_{i}\right] } \\
= & \left(x_{j}-x_{i}\right)+[C(P \backslash\{i\})-C(P \backslash\{j\})] \\
= & \left(\overline{\theta_{j}^{M}}(P, C)-\overline{\theta_{i}^{M}}(P, C)\right)+[C(P \backslash\{i\})-C(P \backslash\{j\})] .
\end{aligned}
$$

On the other hand, by definitions of $\overline{\theta^{M}}, \theta^{M}$,

$$
\begin{aligned}
& \overline{\theta_{i}^{M}}(P, C)-\overline{\theta_{j}^{M}}(P, C) \\
= & \theta_{i}^{M}(P, C)-\theta_{j}^{M}(P, C) \\
= & {[C(P)-C(P \backslash\{i\})]-[C(P)-C(P \backslash\{j\})] } \\
= & C(P \backslash\{j\})-C(P \backslash\{i\}) .
\end{aligned}
$$

By Equations (5) and (6),

$$
\operatorname{ex}\left(P \backslash\{i\}, C, \overline{\theta^{M}}(P, C)\right)=\operatorname{ex}\left(P \backslash\{j\}, C, \overline{\theta^{M}}(P, C)\right),
$$


i.e., $\overline{\theta^{M}}$ satisfies CSE. To verify (2), let $(P, C) \in T G$ and $x=\overline{\theta^{B O}}(P, C)$. For all $i, j \in P$,

$$
\begin{aligned}
& \sum_{S \subseteq P \backslash\{i, j\}} \operatorname{ex}\left(S \cup\{i\}, C, \frac{x}{2^{P \mid-1}}\right)-\sum_{S \subseteq P \backslash\{i, j\}} \operatorname{ex}\left(S \cup\{j\}, C, \frac{x}{2^{|P|-1}}\right) \\
= & \sum_{S \subseteq P \backslash\{i, j\}}\left[C(S \cup\{i\})-\frac{|x| s \cup\{i\}}{2^{|P|-1}}\right]-\sum_{S \subseteq P \backslash\{i, j\}}\left[C(S \cup\{j\})-\frac{|x| S \cup\{\}\}}{2^{|P|-1}}\right] \\
= & {\left[\sum_{S \subseteq P \backslash\{i, j\}} C(S \cup\{i\})\right]-\frac{x_{i}}{2}-\left[\sum_{S \subseteq P \backslash\{i, j\}} C(S \cup\{j\})\right]+\frac{x_{j}}{2} } \\
= & \frac{1}{2}\left(x_{j}-x_{i}\right)+\sum_{S \subseteq P \backslash\{i, j\}}[C(S \cup\{i\})-C(S \cup\{j\})] \\
= & \frac{1}{2}\left(\overline{\theta_{j}^{B O}}(P, C)-\overline{\theta^{B O}}{ }_{i}\right)+\sum_{S \subseteq P \backslash\{i, j\}}[C(S \cup\{i\})-C(S \cup\{j\})] .
\end{aligned}
$$

On the other hand, by definitions of $\overline{\theta^{B O}}, \theta^{B O}$,

$$
\begin{aligned}
& \overline{\theta_{i}^{B O}}(P, C)-\overline{\theta_{j}^{B O}}(P, C) \\
= & \theta_{i}^{B O}(P, C)-\theta_{j}^{B O}(P, C) \\
= & {\left[\sum_{S \subseteq P \backslash\{i\}}(C(S \cup\{i\})-C(S)]-\left[\sum_{S \subseteq P \backslash\{j\}}(C(S \cup\{j\})-C(S)]\right.\right.} \\
= & {\left[\sum_{S \subseteq P \backslash\{i, j\}}(C(S \cup\{i, j\})-C(S \cup\{j\}))+\sum_{S \subseteq P \backslash\{i, j\}}(C(S \cup\{i\})-C(S))\right] } \\
& -\left[\sum_{S \subseteq P \backslash\{i, j\}} C(S \cup\{i, j\})-C(S \cup\{i\})+\sum_{S \subseteq P \backslash\{i, j\}}(C(S \cup\{j\})-C(S))\right] \\
= & 2 \sum_{S \subseteq P \backslash\{i, j\}}[C(S \cup\{i\})-C(S \cup\{j\})] .
\end{aligned}
$$

By Equations (7) and (8),

$$
\sum_{S \subseteq P \backslash\{i, j\}} \operatorname{ex}\left(S \cup\{i\}, C, \frac{\overline{\theta^{B O}}(P, C)}{2^{|P|-1}}\right)=\sum_{S \subseteq P \backslash\{i, j\}} \operatorname{ex}\left(S \cup\{j\}, C, \frac{\overline{\theta^{B O}}(P, C)}{2^{|P|-1}}\right),
$$

i.e., $\overline{\theta^{B O}}$ satisfies ASE. To verify (3), let $(P, C) \in T G$ and $x=\overline{\theta^{B C}}(P, C)$. For all $i, j \in P$,

$$
\begin{aligned}
& \sum_{S \subseteq P \backslash\{i, j\}} \operatorname{ex}(S \cup\{i\}, C, x)-\sum_{S \subseteq P \backslash\{i, j\}} \operatorname{ex}(S \cup\{j\}, C, x) \\
= & \sum_{S \subseteq P \backslash\{i, j\}}\left[C(S \cup\{i\})-|x|_{S \cup\{i\}}\right]-\sum_{S \subseteq P \backslash\{i, j\}}\left[C(S \cup\{j\})-|x|_{S \cup\{j\}}\right] \\
= & \left(\sum_{S \subseteq P \backslash\{i, j\}} C(S \cup\{i\})\right)-2^{|P|-2} \cdot x_{i}-\left(\sum_{S \subseteq P \backslash\{i, j\}} C(S \cup\{j\})\right)+2^{|P|-2} \cdot x_{j} \\
= & 2^{|P|-2} \cdot\left(x_{j}-x_{i}\right)-\sum_{S \subseteq P \backslash\{i, j\}}[C(S \cup\{i\})-C(S \cup\{j\})] .
\end{aligned}
$$

On the other hand, by definitions of $\overline{\theta^{B C}}, \theta^{B C}$,

$$
\begin{aligned}
& \overline{\theta_{i}^{B C}}(P, C)-\overline{\theta_{j}^{B C}}(P, C) \\
= & \theta_{i}^{B C}(P, C)-\theta_{j}^{B C}(P, C) \\
= & \frac{1}{2^{|P|-1}} \cdot\left[\left(\sum_{S \subseteq P \backslash\{i\}}(C(S \cup\{i\})-C(S))-\left(\sum_{S \subseteq P \backslash\{j\}}(C(S \cup\{j\})-C(S))\right]\right.\right. \\
= & \frac{1}{2^{|P|-1}} \cdot \sum_{S \subseteq P \backslash\{i, j\}} 2 \cdot[C(S \cup\{i\})-C(S \cup\{j\})] \\
= & \frac{1}{2^{|P|-2}} \cdot \sum_{S \subseteq P \backslash\{i, j\}}[C(S \cup\{i\})-C(S \cup\{j\})] .
\end{aligned}
$$

By Equations (9) and (10),

$$
\sum_{S \subseteq P \backslash\{i, j\}} \operatorname{ex}\left(S \cup\{i\}, C, \overline{\theta^{B C}}(P, C)\right)=\sum_{S \subseteq P \backslash\{i, j\}} \operatorname{ex}\left(S \cup\{j\}, C, \overline{\theta^{B C}}(P, C)\right),
$$


i.e., $\overline{\theta^{B C}}$ satisfies SSE.

The following theorem presents that the three indexes could be characterized by means of corresponding symmetry respectively.

\section{Theorem 1.}

1. The index $\overline{\theta^{M}}$ is the only index satisfying EFF and CSE.

2. The index $\overline{\theta^{B O}}$ is the only index satisfying EFF and ASE.

3. The index $\overline{\theta^{B C}}$ is the only index satisfying EFF and SSE.

Proof. Let $\kappa$ be an index satisfying EFF. To verify $(1)$, let $(P, C) \in T G$. Assume that $\kappa$ satisfies CSE. If $|P|=1$, it is trivial by EFF of $\kappa$ and $\overline{\theta^{M}}$. Assume that $|P| \geq 2$ and $i, j \in P$. By CSE of $\kappa$,

$$
\begin{aligned}
& \operatorname{ex}(P \backslash\{i\}, C, \kappa(P, C))=\operatorname{ex}(P \backslash\{j\}, C, \kappa(P, C)) \\
\Leftrightarrow & {\left[C(P \backslash\{i\})-|\kappa(P, C)|_{P \backslash\{i\}}\right]=\left[C(P \backslash\{j\})-|\kappa(P, C)|_{P \backslash\{j\}}\right] } \\
\Leftrightarrow & {\left[C(P \backslash\{i\})-\kappa_{j}(P, C)\right]=\left[C(P \backslash\{j\})-\kappa_{i}(P, C)\right] } \\
\Leftrightarrow & \kappa_{i}(P, C)-\kappa_{j}(P, C)=C(P \backslash\{i\})-C(P \backslash\{j\}) .
\end{aligned}
$$

By Equations (6) and (11),

$$
\kappa_{i}(P, C)-\kappa_{j}(P, C)=\overline{\theta_{i}^{M}}(P, C)-\overline{\theta_{j}^{M}}(P, C) .
$$

Based on Equation (12) and EFF of $\overline{\theta^{M}}$ and $\kappa$,

$$
\begin{aligned}
& \left.\kappa_{i}(P, C)-\kappa_{j}(P, C)=\overline{\theta_{i}^{M}}(P, C)-\overline{\theta_{j}^{M}}(P, C)\right) \\
\Leftrightarrow & \sum_{j \in P}\left[\kappa_{i}(P, C)-\kappa_{j}(P, C)\right]=\sum_{j \in P}\left[\overline{\theta_{i}^{M}}(P, C)-\overline{\theta_{j}^{M}}(P, C)\right] \\
\Leftrightarrow & |P| \cdot \kappa_{i}(P, C)-C(P)=|P| \cdot \overline{\theta_{i}^{M}}(P, C)-C(P) \\
\Leftrightarrow & \kappa_{i}(P, C)=\overline{\theta_{i}^{M}}(P, C),
\end{aligned}
$$

i.e., $\kappa_{i}(P, C)=\overline{\theta_{i}^{M}}(P, C)$ for all $i \in P$. To verify (2), let $(P, C) \in T G$. Assume that $\kappa$ satisfies ASE. If $|P|=1$, it is trivial by EFF of $\kappa$ and $\overline{\theta^{B O}}$. Assume that $|P| \geq 2$ and $i, j \in P$. By ASE of $\kappa$,

$$
\begin{aligned}
& \sum_{S \subseteq P \backslash\{i, j\}} \operatorname{ex}\left(S \cup\{i\}, C, \frac{\kappa(P, C)}{2^{|P|-1}}\right)=\sum_{S \subseteq P \backslash\{i, j\}} \operatorname{ex}\left(S \cup\{j\}, C, \frac{\kappa(P, C)}{2^{P \mid-1}}\right) \\
\Leftrightarrow & \sum_{S \subseteq P \backslash\{i, j\}}\left[C(S \cup\{i\})-\frac{|\kappa(P, C)|}{2^{|P|-1}}\right]=\sum_{S \subseteq P \backslash\{i, j\}}\left[C(S \cup\{j\})-\frac{|\kappa(P, C)|}{2^{|P|-1}}\right] \\
\Leftrightarrow & {\left[\sum_{S \subseteq P \backslash\{i, j\}} C(S \cup\{i\})\right]-\frac{\kappa_{i}(P, C)}{2}=\left[\sum_{S \subseteq P \backslash\{i, j\}} C(S \cup\{j\})\right]-\frac{\kappa_{j}(P, C)}{2} } \\
\Leftrightarrow & \kappa_{i}(P, C)-\kappa_{j}(P, C)=2 \sum_{S \subseteq P \backslash\{i, j\}}[C(S \cup\{i\})-C(S \cup\{j\})] .
\end{aligned}
$$

By Equations (8) and (13),

$$
\kappa_{i}(P, C)-\kappa_{j}(P, C)=\overline{\theta_{i}^{B O}}(P, C)-\overline{\theta_{j}^{B O}}(P, C) .
$$


Based on Equation (14) and EFF of $\overline{\theta^{B O}}$ and $\kappa$,

$$
\begin{aligned}
& \left.\kappa_{i}(P, C)-\kappa_{j}(P, C)=\overline{\theta_{i}^{B O}}(P, C)-\overline{\theta_{j}^{B O}}(P, C)\right) \\
\Leftrightarrow & \sum_{j \in P}\left[\kappa_{i}(P, C)-\kappa_{j}(P, C)\right]=\sum_{j \in P}\left[\overline{\theta_{i}^{B O}}(P, C)-\overline{\theta_{j}^{B O}}(P, C)\right] \\
\Leftrightarrow & |P| \cdot \kappa_{i}(P, C)-C(P)=|P| \cdot \overline{\theta_{i}^{B O}}(P, C)-C(P) \\
\Leftrightarrow & \kappa_{i}(P, C)=\overline{\theta_{i}^{B O}}(P, C),
\end{aligned}
$$

i.e., $\kappa_{i}(P, C)=\overline{\theta_{i}^{B O}}(P, C)$ for all $i \in P$. To verify (3), let $(P, C) \in T G$. Assume that $\kappa$ satisfies SSE. If $|P|=1$, it is trivial by EFF of $\kappa$ and $\overline{\theta^{B C}}$. Assume that $|P| \geq 2$ and $i, j \in P$. By SSE of $\kappa$,

$$
\begin{aligned}
& \sum_{S \subseteq P \backslash\{i, j\}} \operatorname{ex}(S \cup\{i\}, C, \kappa(P, C))=\sum_{S \subseteq P \backslash\{i, j\}} \operatorname{ex}(S \cup\{j\}, C, \kappa(P, C)) \\
\Leftrightarrow & \sum_{S \subseteq P \backslash\{i, j\}}\left[C(S \cup\{i\})-|\kappa(P, C)|_{S \cup\{i\}}\right]=\sum_{S \subseteq P \backslash\{i, j\}}\left[C(S \cup\{j\})-|\kappa(P, C)|_{S \cup\{j\}}\right] \\
\Leftrightarrow & \left(\sum_{S \subseteq P \backslash\{i, j\}} C(S \cup\{i\})\right)-2^{|P|-2} \cdot \kappa_{i}(P, C)=\left(\sum_{S \subseteq P \backslash\{i, j\}} C(S \cup\{j\})\right)-2^{|P|-2} \cdot \kappa_{j}(P, C) \\
\Leftrightarrow & 2^{|P|-2} \cdot\left(\kappa_{j}(P, C)-\kappa_{i}(P, C)\right)=\sum_{S \subseteq P \backslash\{i, j\}}[C(S \cup\{i\})-C(S \cup\{j\})] .
\end{aligned}
$$

By Equations (10) and (15),

$$
\kappa_{i}(P, C)-\kappa_{j}(P, C)=\overline{\theta_{i}^{B C}}(P, C)-\overline{\theta_{j}^{B C}}(P, C) .
$$

Based on Equation (16) and EFF of $\overline{\theta^{B C}}$ and $\kappa$,

$$
\begin{aligned}
& \left.\kappa_{i}(P, C)-\kappa_{j}(P, C)=\overline{\theta_{i}^{B C}}(P, C)-\overline{\theta_{j}^{B C}}(P, C)\right) \\
\Leftrightarrow & \sum_{j \in P}\left[\kappa_{i}(P, C)-\kappa_{j}(P, C)\right]=\sum_{j \in P}\left[\overline{\theta_{i}^{B C}}(P, C)-\overline{\theta_{j}^{B C}}(P, C)\right] \\
\Leftrightarrow & |P| \cdot \kappa_{i}(P, C)-C(P)=|P| \cdot \overline{\theta_{i}^{B C}}(P, C)-C(P) \\
\Leftrightarrow & \kappa_{i}(P, C)=\overline{\theta_{i}^{B C}}(P, C),
\end{aligned}
$$

i.e., $\kappa_{i}(P, C)=\overline{\theta_{i}^{B C}}(P, C)$ for all $i \in P$.

\section{Dynamic Processes}

Based on corresponding symmetry, this section proposes dynamic processes that lead the participants to these indexes, starting from a payoff vector generated from arbitrary efficient indexes.

A dynamic process mainly refers to a modification process in which unsatisfactory outcomes gradually trend towards balanced outcomes. Subsequently, the power indexes that produce balanced outcomes are generally assessed by using axiomatic characterization to validate their uniqueness and verify that they meet fair, just, and widely accepted axioms. According to the outcomes of a literature review and the axiomatic characterization results of this study, these power indexes were the solution concept that were individually fair, just, and widely accepted. Stearns [17] was the first to introduce the concept of dynamic processes. Maschler and Owen [13] adopted the reduction concept of "reallocate unsatisfactory outcomes" proposed by Hart and incorporated the variance derived from modification and reallocation into the dynamic process of the Shapley value. Hwang [7] and Hwang and Liao [4,5] combined the differences between process involvement and allocation outcomes with different participatory behaviors to create a set of excess concepts for a compensation vector. The concepts were then applied to develop different dynamic processes for EANSC, EBCI, and EBOI. 
Based on the aforementioned outcomes of dynamic processes, this article first determines the differences between process involvement and outcomes and adopted different participatory behaviors to develop a set of excess concepts related to power index. Then, this article examines different symmetry properties and developed corresponding modification functions to gradually adjust the asymmetrical outcomes to achieve symmetry. The axiomatic characterization results indicated that the efficient outcomes that achieve symmetry are consistent with the outcomes derived from EANSC, EBCI, and EBOI.

Let $(P, C) \in T G$ and $\kappa$ be an efficient index. Define the modifying functions $M^{M}=$ $\left(M_{i}^{M}\right)_{i \in P}, M^{B O}=\left(M_{i}^{B O}\right)_{i \in P}, M^{B C}=\left(M_{i}^{B C}\right)_{i \in P}$ as follows. For all $i \in P, x=\kappa(P, C)$ and

$$
\begin{aligned}
& M_{i}^{M}(x)=x_{i}+\lambda \sum_{j \in P \backslash\{i\}}(\operatorname{ex}(P \backslash\{j\}, C, x)-e x(P \backslash\{i\}, C, x)), \\
& M_{i}^{B O}(x)=x_{i}+\lambda \sum_{j \in P \backslash\{i\}}\left(\sum_{Q \subseteq P \backslash\{i, j\}}\left[\operatorname{ex}\left(Q \cup\{i\}, C, \frac{x}{2^{|P|-1}}\right)-e x\left(Q \cup\{j\}, C, \frac{x}{2^{|P|-1}}\right)\right]\right), \\
& M_{i}^{B C}(x)=x_{i}+\lambda \sum_{j \in P \backslash\{i\}}\left(\frac{1}{2^{|P|-2}} \sum_{Q \subseteq P \backslash\{i, j\}}[\operatorname{ex}(Q \cup\{i\}, C, x)-\operatorname{ex}(Q \cup\{j\}, C, x)]\right) .
\end{aligned}
$$

Based on the results of Theorem 1, it is obvious that $M^{M}\left(\overline{\theta^{M}}(P, C)\right)=\overline{\theta^{M}}(P, C)$, $M^{B O}\left(\overline{\theta^{B O}}(P, C)\right)=\overline{\theta^{B O}}(P, C), M^{B C}\left(\overline{\theta^{B C}}(P, C)\right)=\overline{\theta^{B C}}(P, C)$.

The modifying functions measure the sum of the differences of these different excesses between any participant and any other participant respectively. A coefficient is further adopted as the modifier to adjust the original outcomes.

The following results show that these modifying functions are well-defined, i.e., if $\sum_{i \in P} \kappa_{i}(P, C)=C(P)$, then $\sum_{i \in P} C_{i}(\kappa(P, C))=C(P)$, where $C=M^{M}, M^{B O}, M^{B C}$. These results play key roles to prove the necessary convergence results.

Lemma 2. Let $(P, C) \in T G$ and $\kappa$ be an efficient index. Then for all $i \in P$,

1. $\sum_{j \in P \backslash\{i\}}(\operatorname{ex}(P \backslash\{j\}, C, \kappa(P, C))-e x(P \backslash\{i\}, C, \kappa(P, C)))=|P|\left(\overline{\theta_{i}^{M}}(P, C)-\kappa_{i}(P, C)\right)$; $\sum_{i \in P} \sum_{j \in P \backslash\{i\}}(\operatorname{ex}(P \backslash\{j\}, C, \kappa(P, C))-\operatorname{ex}(P \backslash\{i\}, C, \kappa(P, C)))=0$.

2. $\sum_{j \in P \backslash\{i\}}\left(\sum_{Q \subseteq P \backslash\{i, j\}}\left[\operatorname{ex}\left(Q \cup\{i\}, C, \frac{\kappa(P, C)}{2^{|P|-1}}\right)-\operatorname{ex}\left(Q \cup\{j\}, C, \frac{\kappa(P, C)}{2^{|P|-1}}\right)\right]\right)=\frac{|P|}{2}\left(\overline{\theta_{i}^{B C}}(P, C)-\right.$ $\left.\kappa_{i}(P, C)\right)$;

$$
\sum_{i \in P} \sum_{j \in P \backslash\{i\}}\left(\sum_{Q \subseteq P \backslash\{i, j\}}\left[\operatorname{ex}\left(Q \cup\{i\}, C, \frac{\kappa(P, C)}{2^{|P|-1}}\right)-\operatorname{ex}\left(Q \cup\{j\}, C, \frac{\kappa(P, C)}{2^{|P|-1}}\right)\right]\right)=0 .
$$

3. $\sum_{j \in P \backslash\{i\}} \frac{1}{\frac{1}{|P|-2}_{Q \subseteq P \backslash\{i, j\}}} \sum[\operatorname{ex}(Q \cup\{i\}, C, \kappa(P, C))-\operatorname{ex}(Q \cup\{j\}, C, \kappa(P, C))]$

$$
\begin{aligned}
& =|P|\left(\overline{\theta_{i}^{B O}}(P, C)-\kappa_{i}(P, C)\right) ; \\
& \sum_{i \in P} \sum_{j \in P \backslash\{i\}}\left(\frac{1}{2^{|P|-2}} \sum_{Q \subseteq P \backslash\{i, j\}}[\operatorname{ex}(Q \cup\{i\}, C, \kappa(P, C))-e x(Q \cup\{j\}, C, \kappa(P, C))]\right)=0 .
\end{aligned}
$$

Proof. Let $(P, C) \in T G, i, j \in P, \kappa$ be an efficient index and $x=\kappa(P, C)$. To verify (1),

$$
\begin{aligned}
& \sum_{j \in P \backslash\{i\}}(\operatorname{ex}(P \backslash\{j\}, C, x)-\operatorname{ex}(P \backslash\{i\}, C, x)) \\
= & \sum_{j \in P \backslash\{i\}}\left(C(P \backslash\{j\})-|x|_{P \backslash\{j\}}-C(P \backslash\{i\})+|x|_{P \backslash\{i\}}\right) \\
= & \sum_{j \in P \backslash\{i\}}\left(C(P \backslash\{j\})-C(P \backslash\{i\})-x_{i}+x_{j}\right) .
\end{aligned}
$$


By Equations (6) and (17),

$$
\begin{aligned}
& \sum_{j \in P \backslash\{i\}}(\operatorname{ex}(P \backslash\{j\}, C, x)-e x(P \backslash\{i\}, C, x)) \\
= & \sum_{j \in P \backslash\{i\}}\left(\overline{\theta_{i}^{M}}(P, C)-\overline{\theta_{j}^{M}}(P, C)-x_{i}+x_{j}\right) \\
= & \left((|P|-1) \overline{\theta_{i}^{M}}(P, C)-\overline{\theta^{M}}(P, C)(P \backslash\{i\})-(|P|-1) x_{i}+x(P \backslash\{i\})\right) \\
= & \left(|P| \overline{\theta_{i}^{M}}(P, C)-C(P)-|P| x_{i}+C(P)\right) \\
& \left(\text { by EFF of } \overline{\theta^{M}} \text { and } \kappa\right) \\
= & |P|\left(\overline{\theta_{i}^{M}}(P, C)-x_{i}\right) .
\end{aligned}
$$

Moreover,

$$
\begin{aligned}
& \sum_{i \in P} \sum_{j \in P \backslash\{i\}}(\operatorname{ex}(P \backslash\{j\}, C, x)-\operatorname{ex}(P \backslash\{i\}, C, x)) \\
= & \sum_{i \in P}|P|\left(\overline{\theta_{i}^{M}}(P, C)-x_{i}\right) \\
= & |P|\left(\sum_{i \in P} \overline{\theta_{i}^{M}}(P, C)-\sum_{i \in P} x_{i}\right) \\
= & |P|(C(P)-C(P)) \\
& \left(\text { by EFF of } \overline{\theta^{M}} \text { and } \kappa\right) \\
= & 0 .
\end{aligned}
$$

To verify (2),

$$
\begin{aligned}
& \sum_{j \in P \backslash\{i\}}\left(\sum_{Q \subseteq P \backslash\{i, j\}}\left[\operatorname{ex}\left(Q \cup\{i\}, C, \frac{x}{2^{P \mid-1}}\right)-\operatorname{ex}\left(Q \cup\{j\}, C, \frac{x}{2^{|P|-1}}\right)\right]\right) \\
= & \sum_{j \in P \backslash\{i\}}\left(\sum_{Q \subseteq P \backslash\{i, j\}}\left[C(Q \cup\{i\})-\frac{|x|_{Q \cup\{i\}}}{2^{|P|-1}}-C(Q \cup\{j\})+\frac{|x|_{Q \cup\{j\}}}{2^{P \mid-1}}\right]\right) \\
= & \sum_{j \in P \backslash\{i\}}\left(\left[\sum_{Q \subseteq P \backslash\{i, j\}}[C(Q \cup\{i\})-C(Q \cup\{j\})]\right]-\frac{1}{2}\left[x_{i}-x_{j}\right]\right) .
\end{aligned}
$$

By Equations (8) and (18),

$$
\begin{aligned}
& \sum_{j \in P \backslash\{i\}}\left(\sum_{Q \subseteq P \backslash\{i, j\}}\left[\operatorname{ex}\left(Q \cup\{i\}, C, \frac{x}{2^{|P|-1}}\right)-e x\left(Q \cup\{j\}, C, \frac{x}{2^{|P|-1}}\right)\right]\right) \\
= & \frac{1}{2} \sum_{j \in P \backslash\{i\}}\left(\overline{\theta_{i}^{B O}}(P, C)-\overline{\theta_{j}^{B O}}(P, C)-x_{i}+x_{j}\right) \\
= & \frac{1}{2}\left((|P|-1) \overline{\theta_{i}^{B O}}(P, C)-\overline{\theta^{B O}}(P, C)(P \backslash\{i\})-(|P|-1) x_{i}+x(P \backslash\{i\})\right) \\
= & \frac{1}{2}\left(|P| \overline{\theta_{i}^{B O}}(P, C)-C(P)-|P| x_{i}+C(P)\right) \\
& \left(\text { by EFF of } \overline{\theta^{B O}} \text { and } \kappa\right) \\
= & \frac{P P \mid}{2}\left(\overline{\theta_{i}^{B O}}(P, C)-x_{i}\right) .
\end{aligned}
$$

Moreover,

$$
\begin{aligned}
& \sum_{i \in P} \sum_{j \in P \backslash\{i\}}\left(\sum_{Q \subseteq P \backslash\{i, j\}}\left[\operatorname{ex}\left(Q \cup\{i\}, C, \frac{x}{2^{|P|-1}}\right)-e x\left(Q \cup\{j\}, C, \frac{x}{2^{|P|-1}}\right)\right]\right) \\
= & \sum_{i \in P} \frac{|P|}{2}\left(\overline{\theta_{i}^{B O}}(P, C)-x_{i}\right) \\
= & \frac{|P|}{2}\left(\sum_{i \in P} \overline{\theta_{i}^{B O}}(P, C)-\sum_{i \in P} x_{i}\right) \\
= & \frac{|P|}{2}(C(P)-C(P)) \\
= & \quad\left(\text { by EFF of } \overline{\theta^{B O}} \text { and } \kappa\right) \\
= &
\end{aligned}
$$


To verify (3),

$$
\begin{aligned}
& \sum_{j \in P \backslash\{i\}}\left(\frac{1}{2^{P P \mid-2}} \quad \sum_{Q \subseteq P \backslash\{i, j\}}[\operatorname{ex}(Q \cup\{i\}, C, x)-e x(Q \cup\{j\}, C, x)]\right) \\
= & \sum_{j \in P \backslash\{i\}}\left(\frac{1}{2^{|P|-2}} \sum_{Q \subseteq P \backslash\{i, j\}}\left[C(Q \cup\{i\})-|x|_{Q \cup\{i\}}-C(Q \cup\{j\})+|x|_{Q \cup\{j\}])}\right]\right. \\
= & \sum_{j \in P \backslash\{i\}}\left(\frac{1}{2^{|P|-2}} \sum_{Q \subseteq P \backslash\{i, j\}}\left[C(Q \cup\{i\})-C(Q \cup\{j\})-x_{i}+x_{j}\right]\right) \\
= & \sum_{j \in P \backslash\{i\}}\left(\left(\frac{1}{2^{|P|-2}} \sum_{Q \subseteq P \backslash\{i, j\}}[C(Q \cup\{i\})-C(Q \cup\{j\})]\right)-x_{i}+x_{j}\right) .
\end{aligned}
$$

By Equations (10) and (19),

$$
\begin{aligned}
& \sum_{j \in P \backslash\{i\}}\left(\frac{1}{2^{|P|-2}} \sum_{Q \subseteq P \backslash\{i, j\}}[\operatorname{ex}(Q \cup\{i\}, C, x)-\operatorname{ex}(Q \cup\{j\}, C, x)]\right) \\
= & \sum_{j \in P \backslash\{i\}}\left(\overline{\theta_{i}^{B C}}(P, C)-\overline{\theta_{j}^{B C}}(P, C)-x_{i}+x_{j}\right) \\
= & \left((|P|-1) \overline{\theta_{i}^{B C}}(P, C)-\overline{\theta^{B C}}(P, C)(P \backslash\{i\})-(|P|-1) x_{i}+x(P \backslash\{i\})\right) \\
= & \left(|P| \overline{\theta_{i}^{B C}}(P, C)-C(P)-|P| x_{i}+C(P)\right) \\
& \left(\text { by EFF of } \overline{\theta^{B C}} \text { and } \kappa\right) \\
= & |P|\left(\overline{\theta_{i}^{B C}}(P, C)-x_{i}\right) .
\end{aligned}
$$

Moreover,

$$
\begin{aligned}
& \sum_{i \in P} \sum_{j \in P \backslash\{i\}}\left(\sum_{Q \subseteq P \backslash\{i, j\}}[\operatorname{ex}(Q \cup\{i\}, C, x)-\operatorname{ex}(Q \cup\{j\}, C, x)]\right) \\
= & \sum_{i \in P}|P|\left(\overline{\theta_{i}^{B C}}(P, C)-x_{i}\right) \\
= & |P|\left(\sum_{i \in P} \overline{\theta_{i}^{B C}}(P, C)-\sum_{i \in P} x_{i}\right) \\
= & |P|(C(P)-C(P)) \\
& \left(\text { by EFF of } \overline{\theta^{B C}} \text { and } \kappa\right) \\
= & 0 .
\end{aligned}
$$

The proof is completed.

Let $(P, C) \in T G, \kappa$ be an efficient index and $x=\kappa(P, C)$. Define $\left\{x_{M^{M}}^{q}\right\}_{q=1}^{\infty},\left\{x_{M^{B O}}^{q}\right\}_{q=1}^{\infty}$ and $\left\{x_{M^{B C}}^{q}\right\}_{q=1}^{\infty}$ related to the modifying functions $M^{M}, M^{B O}$, and $M^{B C}$ as follows. For all $q \in \mathbb{N}$,

$$
\begin{aligned}
& x_{M^{M}}^{0}=x, x_{M^{M}}^{1}=M^{M}\left(x_{M^{M}}^{0}\right), x_{M^{M}}^{2}=M^{M}\left(x_{M^{M}}^{1}\right), \cdots, x_{M^{M}}^{q}=M^{M}\left(x_{M^{M}}^{q-1}\right) ; \\
& x_{M^{B O}}^{0}=x, x_{M^{B O}}^{1}=M^{B O}\left(x_{M^{B O}}^{0}\right), x_{M^{B O}}^{2}=M^{B O}\left(x_{M^{B O}}^{1}\right), \cdots, x_{M^{B O}}=M^{B O}\left(x_{M^{B O}}^{q-1}\right) ; \\
& x_{M^{B C}}^{0}=x, x_{M^{B C}}^{1}=M^{B C}\left(x_{M^{B C}}^{0}\right), x_{M^{B C}}^{2}=M^{B C}\left(x_{M^{B C}}^{1}\right), \cdots, x_{M^{B C}}^{q}=M^{B C}\left(x_{M^{B C}}^{q-1}\right) .
\end{aligned}
$$

The results below show the range of coefficients that enables efficient outcomes to gradually approach the three power indexes by using the three modifying functions.

Theorem 2. Let $(P, C) \in T G, \kappa$ be an efficient index and $x=\kappa(P, C)$.

1. If $0<\lambda<\frac{2}{|P|}$, then $\left\{x_{M^{M}}^{q}\right\}_{q=1}^{\infty}$ converges to $\overline{\theta^{M}}(P, C)$.

2. If $0<\lambda<\frac{4}{|P|}$, then $\left\{x_{M^{B O}}^{q}\right\}_{q=1}^{\infty}$ converges to $\overline{\theta^{B O}}(P, C)$.

3. If $0<\lambda<\frac{2}{|P|}$, then $\left\{x_{M^{B C}}^{q}\right\}_{q=1}^{\infty}$ converges to $\overline{\theta^{B C}}(P, C)$. 
Proof. Let $(P, C) \in T G, \kappa$ be an efficient index and $x=\kappa(P, C)$ and $i \in P$. To verify (1), by definition of $M^{M}$ and Lemma 2,

$$
\begin{aligned}
M_{i}^{M}(x)-x_{i} & =\lambda \sum_{j \in P \backslash\{i\}}(\operatorname{ex}(P \backslash\{j\}, C, x)-\operatorname{ex}(P \backslash\{i\}, C, x)) \\
& =\lambda \cdot|P|\left(\overline{\theta_{i}^{M}}(P, C)-x_{i}\right) .
\end{aligned}
$$

So,

$$
\begin{aligned}
\overline{\theta_{i}^{M}}(P, C)-M_{i}^{M}(x) & =\overline{\theta_{i}^{M}}(P, C)-x_{i}+x_{i}-M_{i}^{M}(x) \\
& =\overline{\theta_{i}^{M}}(P, C)-x_{i}-\lambda \cdot|P|\left(\overline{\theta_{i}^{M}}(P, C)-x_{i}\right) \\
& =(1-\lambda \cdot|P|)\left[\overline{\theta_{i}^{M}}(P, C)-x_{i}\right] .
\end{aligned}
$$

For all $q \in \mathbb{N}$,

$$
\overline{\theta^{M}}(P, C)-x_{M^{M}}^{q}=(1-\lambda \cdot|P|)^{q}\left[\overline{\theta^{M}}(P, C)-x\right] .
$$

If $0<\lambda<\frac{2}{|P|}$, then $-1<(1-\lambda \cdot|P|)<1$ and $\left\{x_{M^{q}}^{q}\right\}_{q=1}^{\infty}$ converges to $\overline{\theta^{M}}(P, C)$. To verify (2), by definition of $M^{B O}$ and Lemma 2,

$$
\begin{aligned}
M_{i}^{B O}(x)-x_{i} & =\lambda \sum_{j \in P \backslash\{i\}}\left(\sum_{Q \subseteq P \backslash\{i, j\}}\left[\operatorname{ex}\left(Q \cup\{i\}, C, \frac{x}{2^{|P|-1}}\right)-e x\left(Q \cup\{j\}, C, \frac{x}{2^{|P|-1}}\right)\right]\right) \\
& =\frac{|P| \cdot \lambda}{2}\left(\overline{\theta_{i}^{B O}}(P, C)-x_{i}\right) .
\end{aligned}
$$

So,

$$
\begin{aligned}
\overline{\theta_{i}^{B O}}(P, C)-M_{i}^{B O}(x) & =\overline{\theta_{i}^{B O}}(P, C)-x_{i}+x_{i}-M_{i}^{B O}(x) \\
& =\overline{\theta_{i}^{B O}}(P, C)-x_{i}-\frac{|P| \cdot \lambda}{2}\left(\overline{\theta_{i}^{B O}}(P, C)-x_{i}\right) \\
& =\left(1-\frac{|P| \cdot \lambda}{2}\right)\left[\overline{\theta_{i}^{B O}}(P, C)-x_{i}\right] .
\end{aligned}
$$

For all $q \in \mathbb{N}$,

$$
\overline{\theta^{B O}}(P, C)-x_{M^{B O}}^{q}=\left(1-\frac{|P| \cdot \lambda}{2}\right)^{q}\left[\overline{\theta^{B O}}(P, C)-x\right] .
$$

If $0<\lambda<\frac{4}{|P|}$, then $-1<\left(1-\frac{|P| \cdot \lambda}{2}\right)<1$ and $\left\{x_{M^{B O}}^{q}\right\}_{q=1}^{\infty}$ converges to $\overline{\theta^{B O}}(P, C)$. To verify (3), by definition of $M^{B C}$ and Lemma 2,

$$
\begin{aligned}
M_{i}^{B C}(x)-x_{i} & =\lambda \sum_{j \in P \backslash\{i\}}\left(\frac{1}{2^{|P|-2}} \sum_{Q \subseteq P \backslash\{i, j\}}[\operatorname{ex}(Q \cup\{i\}, C, x)-\operatorname{ex}(Q \cup\{j\}, C, x)]\right) \\
& =|P| \cdot \lambda\left(\overline{\theta_{i}^{B C}}(P, C)-x_{i}\right) .
\end{aligned}
$$

So,

$$
\begin{aligned}
\overline{\theta_{i}^{B C}}(P, C)-M_{i}^{B C}(x) & =\overline{\theta_{i}^{B C}}(P, C)-x_{i}+x_{i}-M_{i}^{B C}(x) \\
& =\overline{\theta_{i}^{B C}}(P, C)-x_{i}-\lambda \cdot|P|\left(\overline{\theta_{i}^{B C}}(P, C)-x_{i}\right) \\
& =(1-\lambda \cdot|P|)\left[\overline{\theta_{i}^{B C}}(P, C)-x_{i}\right] .
\end{aligned}
$$

For all $q \in \mathbb{N}$,

$$
\begin{gathered}
\overline{\theta^{B C}}(P, C)-x_{M^{B C}}^{q}=(1-\lambda \cdot|P|)^{q}\left[\overline{\theta^{B C}}(P, C)-x\right] . \\
\text { If } 0<\lambda<\frac{2}{|P|} \text {, then }-1<(1-\lambda \cdot|P|)<1 \text { and }\left\{x_{M^{B C}}^{q}\right\}_{q=1}^{\infty} \text { converges to } \overline{\theta^{B C}}(P, C) .
\end{gathered}
$$




\section{Discussions And Conclusions}

\subsection{Discussions}

Based on Example 1, some discussions are given below to illustrate the applications of its related axiomatic and dynamic results for the three indexes.

1. In Theorem 1, the EANSC is proved to be the only index that satisfies EFF and CSE. In a legislative institution, the EANSC is the only power allocation that conforms to the two natures of "complete and proper allocation of power" and "balancing the difference between any two legislative representatives as to their respective participation in the overall sitting". Based on the results of Theorem 2, in a legislative institution, by continuously adjusting the difference between any two legislative representatives as to their respective participation in all professional committee bills, power allocation among all legislative representatives will become gradually closer to the result of power allocation derived from the EANSC.

2. In Theorem 1, the EBOI is proved to be the only index that satisfies EFF and SSE. In a legislative institution, the EBOI is the only power allocation that conforms to the two natures of "complete and proper allocation of power" and "balancing the total difference between any two legislative representatives as to their respective participation in all professional committee bills". Based on the results of Theorem 2, in a legislative institution, by continuously adjusting the total difference between any two legislative representatives as to their respective participation in all professional committee bills, power allocation among all legislative representatives will become gradually closer to the result of power allocation derived from the EBOI.

3. In Theorem 1, the EBCI is proved to be the only index that satisfies EFF and ASE. In a legislative institution, the EBCI is the only power allocation that conforms to the two natures of "complete and proper allocation of power" and "balancing the average difference between any two legislative representatives as to their respective participation in all professional committee bills". Based on the results of Theorem 2, in a legislative institution, by continuously adjusting the average difference between any two legislative representatives as to their respective participation in all professional committee bills, power allocation among all legislative representatives will become gradually closer to the result of power allocation derived from the EBCI.

\subsection{Conclusions}

The purpose of this article was to apply the EANSC, the EBOI and the EBCI in developing different axiomatic characterizations and dynamic processes. The main findings are as follows:

- $\quad$ This article used different participatory behaviors and the differences between process involvement and outcomes to develop three states of symmetry.

- $\quad$ By applying efficiency axiom and the three states of symmetry axiom, this article proposed axiomatic characterizations corresponding to the three power indexes.

- This article further used the the three states of symmetry axiom and related axiomatic characterizations to propose corresponding dynamic processes.

One should also compare the findings of this article with those proposed in previous studies for the EANSC, the EBOI and the EBCI.

- $\quad$ The three states of symmetry axiom proposed in this article were not mentioned in previous studies.

- Moulin [6] introduced the axioms of efficiency, equal treatment for equal, covariance and complement consistency to characterize the EANSC. Inspired by Hart and Mas-Colell [8], Hwang and Liao [4] characterized the EBOI and EBCI by applying different types of consistency and standardness.

- $\quad$ Based on efficiency axiom and the three states of symmetry axiom, different axiomatic characterizations are proposed in this article respectively. 
- The modifying functions used in this article were inspired by the correction function proposed by Maschler and Owen [13]. However, the main differences between the modifying functions used in this article and those proposed by Maschler and Owen [13] are as follows.

- $\quad$ The correction function proposed by Maschler and Owen [13] is based on reduction. - The modifying functions used in this article are based on excess.

- The excess concepts used to develop the modifying functions are inspired by the excess notions due to Hwang [7] and Hwang and Liao [4,5]. However, the main differences between the functions used in this article and those proposed by Hwang [7] and Hwang and Liao $[4,5]$ are as follows.

- Hwang [7] and Hwang and Liao [4,5] adopted payoff vectors when defining the differences between process involvement and outcomes.

- This article determines the differences between process involvement and outcomes and adopted different participatory behaviors to develop a set of excess concepts related to power indexes.

Through the axiomatic and dynamic processes of this article, one could identify another motivation:

- Can other notions be used along with the three power indexes to propose different axiomatic characterizations and dynamic processes?

We believe this is a noteworthy topic of research.

Author Contributions: Conceptualization, Y.-H.L.; methodology, Y.-H.L. and R.-R.H.; validation, Y.H.L.; formal analysis, Y.-H.L. and R.-R.H.; investigation, Y.-H.L.; writing-original draft preparation, Y.-H.L.; writing-review and editing, Y.-H.L. and R.-R.H. All authors have read and agreed to the published version of the manuscript.

Funding: This research received no external funding.

Institutional Review Board Statement: Not applicable.

Informed Consent Statement: Not applicable.

Data Availability Statement: Data available on request due to privacy restrictions. The data presented in this study are available on request from the corresponding author.

Acknowledgments: The authors are very grateful to the Editors and anonymous referees for patience, assistance, suggestions and comments which much improve the paper.

Conflicts of Interest: The authors declare no conflict of interest.

\section{References}

1. Banzhaf, J.F. Weighted voting doesn't work: A mathematical analysis. Rutgers Law Rev. 1965, 19, 317-343.

2. Owen, G. Multilinear extensions and the Banzhaf value. Nav. Res. Logist. Quart 1975, 22, 741-750. [CrossRef]

3. Ransmeier, J.S. The Tennessee Valley Authority; Vanderbilt University Press: Nashville, TN, USA, 1942.

4. Hwang, Y.A.; Liao, Y.H. Consistency and dynamic approach of indexes. Soc. Choice Welf. 2010, 34, 679-694. [CrossRef]

5. Hwang, Y.A.; Liao, Y.H. Alternative formulation and dynamic process for the efficient Banzhaf-Owen index. Oper. Res. Lett. 2017, 45, 60-62. [CrossRef]

6. Moulin, H. The separability axiom and equal-sharing methods. J. Econ. Theory 1985, 36, 120-148. [CrossRef]

7. Hwang, Y.A. An NTU value under complement reduced game. Int. J. Game Theory 2009, 38, 305-324. [CrossRef]

8. Hart, S.; Mas-Colell, A. Potential, value and consistency. Econometrica 1989, 57, 589-614. [CrossRef]

9. Dubey, P.; Shapley, L.S. Mathematical properties of the Banzhaf power index. Math. Oper. Res. 1979, 4, 99-131. [CrossRef]

10. Lehrer, E. An axiomatization of the Banzhaf value. Int. J. Game Theory 1988, 17, 89-99. [CrossRef]

11. Haller, H. Collusion properties of values. Int. J. Game Theory 1994, 23, 261-281. [CrossRef]

12. van den Brink, R.; van der Lann, G. Axiomatizations of the normalized Banzhaf value and the Shapley value. Soc. Choice Welf. 1998, 15, 567-582. [CrossRef]

13. Maschler, M.; Owen, G. The consonant Shapley value for hyperplane games. Int. J. Game Theory 1989, 18, 389-407. [CrossRef]

14. Peleg, B. On the reduced game property and its converse. Int. J. Game Theory 1986, 15, 187-200. [CrossRef] 
15. Shapley, L.S. A value for P-person game. In Distinctions to the Theory of Games II; Kuhn, H.W., Tucker, A.W., Eds.; Princeton Press: Princeton, NJ, USA, 1953; pp. 307-317.

16. Myerson, R. Conference structures and fair allocation rules. Int. J. Game Theory 1980, 9, 169-182. [CrossRef]

17. Stearns, R.E. Convergent Transfer Schemes for P-Person Games. Trans. Amer. Math. Soc. 1968, 134, 449-459. [CrossRef] 